\title{
Further analysis on stability of delayed neural networks via inequality technique
}

Jiayu Wang

Correspondence: jiaywang@163. com

School of Mathematics and Information Sciences, Weifang University, Weifang 261061,

People's Republic of China

\begin{abstract}
In this paper, further analysis on stability of delayed neural networks is presented via the impulsive delay differential inequality, which was obtained by Li in recent publications. Based on the inequality, some new sufficient conditions ensuring global exponential stability of impulsive delay neural networks are derived, and the estimated exponential convergence rates are also obtained. The conditions are less conservative and restrictive than those established in the earlier references. In addition, some numerical examples are given to show the effectiveness of our obtained results.

Keywords: differential inequality, delay; impulse, global exponential stability, convergence rate
\end{abstract}

\section{Introduction and preliminaries}

In recent years, extensive research has been done in neural networks such as Hopfield neural networks, Cohen-Grossberg neural networks, cellular neural networks, and bidirectional associative memory neural networks, because of their potential applications in pattern recognition, image processing, associative memory, and so on, see [1-28]. Recently, a new type of neural networks-impulsive neural networks display a combination of characteristics of both the continuous-time and discrete-time systems, which is an appropriate description of the phenomena of abrupt qualitative dynamical changes of essentially continuous-time systems, see [4,9,13-22]. The stability of impulsive delay neural networks has become an important topic of theoretical studies and has been investigated by many researchers via different approaches, see [9,13-16,20-22] and the references cited therein. For example, Liu et al. [14] obtained some sufficient conditions on global exponential stability by utilizing impulsive delay differential inequality that has been given by Yue et al. [18] for impulsive high-order Hopfield neural networks with time-varying delays as follows:

$$
\left\{\begin{aligned}
C_{i} \frac{\mathrm{d} u_{i}(t)}{\mathrm{d} t}= & -\frac{u_{i}(t)}{R_{i}}+\sum_{j=1}^{n} T_{i j} g_{j}\left(u_{j}\left(t-\tau_{j}(t)\right)\right) \\
& +\sum_{j=1}^{n} \sum_{l=1}^{n} T_{i j l} g_{j}\left(u_{j}\left(t-\tau_{j}(t)\right)\right) \times g_{l}\left(u_{l}\left(t-\tau_{l}(t)\right)\right)+I_{i}, t \neq t_{k}, t \geq t_{0}, \\
\left.\Delta u_{i}\right|_{t=t_{k}}= & \mathrm{d}_{i} u_{i}\left(t_{k}^{-}\right)+\sum_{j=1}^{n} W_{i j} h_{j}\left(u_{j}\left(t_{k}^{-}-\tau_{j}\left(t_{k}^{-}\right)\right)\right) \\
& +\sum_{j=1}^{n} \sum_{l=1}^{n} W_{i j l} h_{j}\left(u_{j}\left(t_{k}^{-}-\tau_{j}\left(t_{k}^{-}\right)\right)\right) \times h_{l}\left(u_{l}\left(t_{k}^{-}-\tau_{l}\left(t_{k}^{-}\right)\right)\right), i \in \Lambda, k \in \mathbb{Z}_{+} \\
u_{i}(s)= & \phi_{i}(s), s \in\left[t_{0}-\tau, t_{0}\right] .
\end{aligned}\right.
$$

(c) 2011 Wang; licensee Springer. This is an Open Access article distributed under the terms of the Creative Commons Attribution License (http://creativecommons.org/licenses/by/2.0), which permits unrestricted use, distribution, and reproduction in any medium, provided the original work is properly cited. 
In $[20,21], \mathrm{Xu}$ and Yang investigated the global exponential stability of impulsive delay neural networks by establishing a delay differential inequality with impulsive initial conditions. The results extend and improve the recent works [23,24]. More recently, Yang et al. [22] investigated the global exponential stability by Lyapunov function and Halanay inequality for impulsive extended BAM type Cohen-Grossberg neural networks with delays and variable coefficients as follows:

$$
\left\{\begin{array}{l}
x_{i}^{\prime}(t)=-a_{i}\left(x_{i}(t)\right)\left[b_{i}\left(x_{i}(t)\right)-\sum_{j=1}^{m} p_{j i} f_{j}\left(y_{j}(t)\right) u_{j}-\sum_{j=1}^{m} r_{j i} f_{j}\left(y_{j}\left(t-\tau_{j i}\right)\right) v_{j}+c_{i}\right], i=1, \ldots, n \\
y_{j}^{\prime}(t)=-a_{j}\left(y_{j}(t)\right)\left[b_{j}\left(y_{j}(t)\right)-\sum_{i=1}^{n} q_{i j} g_{i}\left(x_{i}(t)\right) w_{i}-\sum_{i=1}^{n} s_{i j} g_{i}\left(x_{i}\left(t-\sigma_{i j}\right)\right) e_{i}+d_{j}\right], j=1, \ldots, m \\
x_{i}(s)=\phi_{i}(s), y_{j}(s)=\psi_{j}(s), s \in\left[t_{0}-\tau, t_{0}\right],
\end{array}\right.
$$

where

$$
\begin{array}{ll}
u_{j}=1+\sum_{k=1}^{\infty} \alpha_{j k} \delta\left(t-t_{k}\right), & v_{j}=1+\sum_{k=1}^{\infty} \beta_{j k} \delta\left(t-t_{k}\right), \\
w_{i}=1+\sum_{k=1}^{\infty} \gamma_{i k} \delta\left(t-t_{k}\right), & e_{i}=1+\sum_{k=1}^{\infty} \lambda_{i k} \delta\left(t-t_{k}\right) .
\end{array}
$$

Although some stability conditions for impulsive delay neural networks proposed in [9,14,15,18-22], they have some conservatism to some extent, and there still exists open room for further improvement.

Recently, Li [25] establishes a new impulsive delay differential inequality as follows:

Lemma 1.1. Let $\alpha, \beta$, $r$ and $\tau$ denote nonnegative constants, and function $f \in P C\left(\mathbb{R}, \mathbb{R}_{+}\right)$ satisfies the scalar impulsive differential inequality

$$
\left\{\begin{array}{lc}
D^{+} f(t) \leq-\alpha f(t)+\beta \sup _{t-\tau \leq s \leq t} f(s)+r \int_{0}^{\sigma} k(s) f(t-s) d s, & t \neq t_{k}, t \geq t_{0}, \\
f\left(t_{k}\right) \leq a_{k} f\left(t_{k}^{-}\right)+b_{k} \sup _{t_{k}-\tau \leq s<t_{k}} f(s), & k \in \mathbb{Z}_{+},
\end{array}\right.
$$

where $0<\sigma \leq+\infty, a_{k}, b_{k}, \in \mathbb{R}_{+}, k(\cdot) \in P C\left([0, \sigma], \mathbb{R}_{+}\right)$satisfies $\int_{0}^{\sigma} k(s) e^{\eta_{0} s} d s<\infty$ for some positive constant $\eta_{0}>0$ in the case when $\sigma=+\infty$. Moreover, when $\sigma=+\infty$, the interval $[t-\sigma, t]$ is understood to be replaced by $(-\infty, t]$.

Assume that

(i) $\alpha>\beta+r \int_{0}^{\sigma} k(s) d s$.

(ii) There exist constants $M>0, \eta>0$ such that

$$
\prod_{k=1}^{n} \max \left\{1, \quad a_{k}+b_{k} e^{\lambda \tau}\right\} \leq M e^{\eta\left(t_{n}-t_{0}\right)}, \quad n \in \mathbb{Z}_{+}
$$

where $\lambda \in\left(0, \eta_{0}\right)$ satisfies

$$
\lambda<\alpha-\beta e^{\lambda \tau}-r \int_{0}^{\sigma} k(s) e^{\lambda s} d s .
$$

Then,

$$
f(t) \leq M \sup _{t_{0}-\max \{\sigma, \tau\} \leq s \leq t_{0}} f(s) e^{-(\lambda-\eta)\left(t-t_{0}\right)}, \quad t \geq t_{0},
$$

In particular, it includes the special case: 
Lemma 1.2. Let $\alpha, \beta$ and $\tau$ denote nonnegative constants, $a_{k}, b_{k} \in \mathbb{R}_{+}$, and function $f \in P C\left(\mathbb{R}, \mathbb{R}_{+}\right)$satisfies

$$
\begin{cases}D^{+} f(t) \leq-\alpha f(t)+\beta \sup _{t-\tau \leq s \leq t} f(s), & t \neq t_{k \prime} \\ f\left(t_{k}\right) \leq a_{k} f\left(t_{k^{-}}\right)+b_{k} \sup _{t_{k}-\tau \leq s<t_{k}} f(s), & k \in \mathbb{Z}_{+},\end{cases}
$$

Assume that

(i) $\alpha>\beta \geq 0$.

(ii) There exist constants $M>0, \eta>0$ such that

$$
\prod_{k=1}^{n} \max \left\{1, \quad a_{k}+b_{k} e^{\lambda \tau}\right\} \leq M e^{\eta\left(t_{n}-t_{0}\right)}, \quad n \in \mathbb{Z}_{+},
$$

where $\lambda>0$ satisfies

$$
\lambda<\alpha-\beta e^{\lambda \tau} .
$$

Then

$$
f(t) \leq M \sup _{t_{0}-\tau \leq s \leq t_{0}} f(s) e^{-(\lambda-\eta)\left(t-t_{0}\right)}, \quad t \geq t_{0} .
$$

The purpose of this paper is to improve the results in $[9,14,15,18-22]$ via the above results in Lemma 1.2, which is a special case of [25]. We will derive some new sufficient conditions to ensure the global exponential stability of equilibrium point for impulsive delay Hopfield neural networks (1.1) and BAM type Cohen-Grossberg neural networks (1.2). The main advantages of the obtained exponential stability conditions include:

(I) In $[9,14,15,18,22]$, all of those results require that the time sequence $\left\{t_{k}\right\}$ satisfies $\inf _{k \in \mathbb{Z}_{+}}\left\{t_{k}-t_{k-1}\right\}>\tau \delta, \delta>1$. But this restriction will not be required in our results.

(II) Even for the case $\inf _{k \in \mathbb{Z}_{+}}\left\{t_{k}-t_{k-1}\right\}>\tau$, our results still can be applied to the case not covered in $[19,20]$.

In addition, some illustrative examples are also given to demonstrate the effectiveness of the obtained results.

\section{Global exponential stability analysis for HNNs}

In this section, we will give some new sufficient conditions on the global exponential stability of equilibrium point for the neural network (1.1). The conditions are less restrictive and conservative than that given in [14].

System (1.1) may be rewritten in the following matrices forms:

$$
\left\{\begin{aligned}
C \frac{\mathrm{d} x(t)}{\mathrm{d} t} & =-R^{-1} x(t)+\left(T+\Gamma^{T} T_{H}\right) f(x(t-\tau(t))), \quad t \neq t_{k}, t \geq t_{0}, \\
\Delta x\left(t_{k}\right) & =D x\left(t_{k}^{-}\right)+\left(W+\Lambda^{T} \Xi\right) \varphi\left(x\left(t_{k}^{-}-\tau\left(t_{k}^{-}\right)\right)\right), k \in \mathbb{Z}_{+}, \\
x(s) & =\varphi(s), s \in\left[t_{0}-\tau, t_{0}\right],
\end{aligned}\right.
$$

Remark 2.1. For detail information about (2.1), one may see [14].

Theorem 2.1. Assume that conditions (i), (ii) in Theorem 1 in [14] hold, and

(iii) there exists a constant $\eta>0$ such that

$$
\rho \doteq \max \left\{1, \quad a^{\star}+b^{\star} \exp \{\lambda \tau\}\right\}<\exp \{\lambda \eta\},
$$


where $\eta=\inf _{k \in \mathbb{Z}_{+}}\left\{t_{k}-t_{k-1}\right\}>0$,

$$
a^{\star}=2 \frac{\lambda_{\max }(P)}{\lambda_{\min }(P)}\|(I+D)\|^{2}, \quad b^{\star}=2 \frac{\lambda_{\max }(P)}{\lambda_{\min }(P)} \times \max _{1 \leq i \leq n}\left\{L_{i}^{2}\right\}\left(\|W\|+\left\|\Lambda^{T}\right\|\|\Xi\|\right)^{2},
$$

and $\lambda>0$ satisfies

$$
\lambda \leq a-b \exp \{\lambda \tau\}
$$

Then the equilibrium point of the system (1.1) is globally exponentially stable with the approximate exponential convergence rate $\lambda-\frac{\ln \rho}{\eta}$.

Remark 2.2. For the proofs of Theorems 2.1, we need only to mention a few points, since the rest is the same as in the proofs of Theorems 1 in [14]. First, similarly one may define $V(t)=x^{T}(t) P x(t)$, and it can be deduced that

$$
\left\{\begin{aligned}
\left.D^{+} V(t)\right|_{(2.1)} & \leq-a V(t)+b \sup _{s \in[t-\tau, t]} V(s) \\
V\left(t_{k}\right) & \leq a^{\star} V\left(t_{k}^{-}\right)+b^{\star} \sup _{s \in\left[t_{k}-\tau, t_{k}\right)} V(s)
\end{aligned}\right.
$$

Then using Lemma 1.2 in this paper (replacing Lemma 1 in [14]), Theorem 2.1 can be obtained.

Similarly we can obtain another stability criterion corresponding to Theorem 2 in [14] as follows:

Theorem 2.2. Assume that conditions (i) in Theorem 2 in [14] hold and

(iii) there exists a constant $\eta>0$ such that

$$
\rho \doteq \max \left\{1, \quad a^{*}+b^{*} \exp \{\lambda \tau\}\right\}<\exp \{\lambda \eta\},
$$

where $\eta=\inf _{k \in \mathbb{Z}_{+}}\left\{t_{k}-t_{k-1}\right\}>0$,

$$
a^{*}=\max _{1 \leq i \leq n}\left\{\left|1+d_{i}\right|\right\}, \quad b^{*}=\max _{1 \leq j \leq n}\left\{\sum_{i=1}^{n}\left(\left|W_{i j}\right|+\sum_{i=1}^{n}\left|W_{i j l}+W_{i l j}\right| N_{l}\right) L_{j}\right\},
$$

and $\lambda>0$ satisfies

$$
\lambda \leq a-b \exp \{\lambda \tau\}
$$

Then the equilibrium point of the system (1.1) is globally exponentially stable with the approximate exponential convergence rate $\lambda-\frac{\ln \rho}{\eta}$.

Remark 2.3. In [14], under the assumption that $\inf _{k \in \mathbb{Z}_{+}}\left\{t_{k}-t_{k-1}\right\}>\tau \delta, \delta>1$., Liu et al. obtained some theorems on exponential stability of (1.1). Note that in our theorem 2.1 and 2.2 , we only require that $\inf _{k \in \mathbb{Z}_{+}}\left\{t_{k}-t_{k-1}\right\}>0$. Thus, our results improve the previous findings.

Example 2.1 Consider the three-neuron Hopfield neural network (1.1) with $g_{1}\left(u_{1}\right)=$ $\tanh \left(0.63 u_{1}\right), g_{2}\left(u_{2}\right)=\tanh \left(0.78 u_{2}\right), g_{3}\left(u_{3}\right)=\tanh \left(0.46 u_{3}\right), h_{1}\left(u_{1}\right)=\tanh \left(0.09 u_{1}\right), h_{2}\left(u_{2}\right)$ $=\tanh \left(0.02 u_{2}\right), h_{3}\left(u_{3}\right)=\tanh \left(0.17 u_{3}\right), C=\operatorname{diag}\left(C_{1}, C_{2}, C_{3}\right)=\operatorname{diag}(0.89,0.88,0.53), R$ $=\operatorname{diag}\left(R_{1}, R_{2}, R_{3}\right)=\operatorname{diag}(0.16,0.12,0.03), D=\operatorname{diag}\left(d_{1}, d_{2}, d_{3}\right)=\operatorname{diag}(-0.95,-0.84$, -0.99), $0 \leq \tau_{i}(t) \leq 0.5, i=1,2,3$ and 


$$
\begin{aligned}
& T=\left(T_{i j}\right)_{3 \times 3}=\left[\begin{array}{ccc}
0.19 & 0.35 & 1.29 \\
0.31 & 0.61 & -0.25 \\
0.07 & -0.37 & 0.44
\end{array}\right], T_{1}=\left(T_{1 i j}\right)_{3 \times 3}=\left[\begin{array}{ccc}
0.05 & 0.14 & 0.28 \\
-0.06 & -0.05 & 0.11 \\
-0.24 & -0.06 & -0.09
\end{array}\right], \\
& T_{2}=\left(T_{2 i j}\right)_{3 \times 3}=\left[\begin{array}{ccc}
0.29 & -0.10 & -0.35 \\
023 & -0.14 & 0.25 \\
0.05 & 0.22 & -0.01
\end{array}\right], T_{3}=\left(T_{3 i j}\right)_{3 \times 3}=\left[\begin{array}{ccc}
-0.23 & 0.07 & 0.03 \\
0.09 & -0.02 & -0.19 \\
0.16 & 0.01 & 0.06
\end{array}\right] \text {, } \\
& W=\left(W_{i j}\right)_{3 \times 3}=\left[\begin{array}{ccc}
-0.04 & -0.05 & 0.16 \\
0.19 & -0.17 & -0.02 \\
0.03 & 0.13 & 0.04
\end{array}\right], W_{1}=\left(T_{1 i j}\right)_{3 \times 3}=\left[\begin{array}{ccc}
-0.01 & 0.01 & -0.03 \\
0.08 & -0.09 & 0.07 \\
0.08 & -0.01 & 0.01
\end{array}\right] \text {, } \\
& W_{2}=\left(W_{2 i j}\right)_{3 \times 3}=\left[\begin{array}{ccc}
0.06 & 0 & 0.04 \\
0.04 & -0.07 & 0.07 \\
-0.02 & -0.06 & 0.05
\end{array}\right], W_{3}=\left(T_{3 i j}\right)_{3 \times 3}=\left[\begin{array}{ccc}
0.04 & -0.04 & 0.01 \\
0.02 & 0.05 & -0.05 \\
-0.02 & 0.03 & -0.02
\end{array}\right] \text {. }
\end{aligned}
$$

In this example, similar to [14], one may choose $P=\operatorname{diag}(0.9,0.7,0.8), \varepsilon_{1}=1, \varepsilon_{2}=2$ such that $\Omega<0$ in Theorem 2.1, and that $a=10.2628>2.3814=b$. Also, we can compute that $\rho=1$. Thus, by Theorem 2.1, the equilibrium point of (2.2) is globally exponentially stable with the approximate convergence rate $\lambda$ for $\inf _{k \in \mathbb{Z}_{+}}\left\{t_{k}-t_{k-1}\right\}>0$, where $\lambda>0$ satisfies the inequality: $\lambda \leq 10.2628-2.3814 \mathrm{e}^{\lambda 0.5}$.

Remark 2.4. In [14], Liu et al. obtained that the equilibrium point of (2.2) is globally exponentially stable for $\inf _{k \in \mathbb{Z}_{+}}\left\{t_{k}-t_{k-1}\right\}>0.505$, which was more restrictive and conservative than that of our result. Therefore, the result in this paper is applicable to more conditions.

\section{Global exponential stability analysis for BAM type CGNNs}

In this section, we will reconsider the global exponential stability of impulsive BAM type Cohen-Grossberg neural networks (1.2).

Theorem 3.1. Assume that $\left(H_{1}\right)-\left(H_{3}\right)$ and (i), (ii) in Theorem 2 in [22]hold; moreover, suppose that

(iii) there exists a constant $\eta>0$ such that

$$
M \doteq \max \left\{1, \quad \frac{\bar{a}}{\underline{a}_{k}^{-1}} r^{-\frac{\bar{a}}{a}} R_{k} \exp \{\lambda \tau\}\right\}<\exp \{\lambda \eta\},
$$

where $\eta=\inf _{k \in \mathbb{Z}_{+}}\left\{t_{k}-t_{k-1}\right\}>0$,

$$
\begin{aligned}
& r_{k}=\min _{1 \leq i \leq n, 1 \leq j \leq m}\left\{1-\bar{a} \sum_{j=1}^{m}\left|q_{i j} \gamma_{i k}\right| L_{i}^{g}, \quad 1-\bar{a} \sum_{i=1}^{n}\left|p_{j i} \alpha_{j k}\right| L_{j}^{f}\right\}>0, \\
& R_{k}=\bar{a} r_{k}^{-1} \max _{1 \leq i \leq n, 1 \leq j \leq m}\left\{\sum_{j=1}^{m}\left|s_{i j} \lambda_{i k}\right| L_{i}^{g}, \quad \sum_{i=1}^{n}\left|r_{j i} \beta_{j k}\right| L_{j}^{f}\right\},
\end{aligned}
$$

and $\lambda>0$ satisfies

$$
\lambda \leq k_{1}-k_{2} \exp \{\lambda \tau\}
$$

Then the equilibrium point of the system (1.2) is globally exponentially stable with the approximate exponential convergence rate $\lambda-\frac{\ln M}{\eta}$. 
Proof. Consider Lyapunov function as follows:

$$
V(t)=\sum_{i=1}^{n} \int_{0}^{z_{i}(t)} \frac{S g n s}{\alpha_{i}(s)} d s+\sum_{j=1}^{m} \int_{0}^{\tilde{z}_{i}(t)} \frac{S g n s}{\alpha_{j}(s)} d s .
$$

Then similar to the proof of Theorem 2 in [22], we arrive at

$$
\left\{\begin{aligned}
\left.D^{+} V(t)\right|_{(1.2)} & \leq-k_{1} V(t)+k_{2} \sup _{s \in[t-\tau, t]} V(s) \\
V\left(t_{k}\right) & \leq \frac{\bar{a}}{\underline{a}} r_{k}^{-1} V\left(t_{k}^{-}\right)+\frac{\bar{a}}{\underline{a}} R_{k} \sup _{s \in\left[t_{k}-\tau, t_{k}\right)} V(s)
\end{aligned}\right.
$$

Then by Lemma 1.2, the result holds.

Remark 3.1. In [22], Yang et al. obtained a sufficient condition for global asymptotic stability of (1.2), which assumes that $\inf _{k \in \mathbb{Z}_{+}}\left\{t_{k}-t_{k-1}\right\}>\tau \delta, \delta>1$, while ours do not impose this restriction.

Example 3.1. Consider the following extended BAM neural networks:

$$
\left\{\begin{array}{l}
x^{\prime}(t)=-(3+\cos x(t))\left[x(t)-\frac{1}{10} \cos t \sin \gamma(t) u_{k}-\frac{1}{100} \sin t \sin (\gamma(t)-18) v_{k}-\frac{\pi}{2}\right], \\
\gamma^{\prime}(t)=-(1+\sin \gamma(t))\left[\gamma(t)-\frac{1}{10} \sin t \cos x(t) w_{k}-\frac{1}{100} \cos t \cos (x(t)-16) e_{k}-\pi\right],
\end{array}\right.
$$

where $u_{k}=w_{k}=v_{k}=e_{k}=1+(-1)^{k} \delta\left(t-t_{k}\right)$, the impulse times $t_{k}$ satisfy $0 \leq t_{0}<t_{1}<$ $<t_{k}<\ldots, \lim _{k \rightarrow+\infty} t_{k}=+\infty$ and $\inf _{k \in \mathbb{Z}_{+}}\left\{t_{k}-t_{k-1}\right\}=16$. Let $\tau=18$.

By simple calculation, we can obtain $k_{1}=\frac{9}{10}, k_{2}=\frac{1}{25}, \quad r_{k}=\frac{3}{5}, \quad R_{k}=\frac{1}{15}$, $M \doteq \max \left\{1, \quad \frac{\bar{a}}{a} r_{k}^{-1}+\frac{\bar{a}}{\underline{a}} R_{k} \exp \{\lambda \tau\}\right\}=\frac{20}{3}+\frac{4}{15} \exp \{18 \lambda\}$, where $\lambda>0$ satisfies the inequality: $\lambda \leq \frac{9}{10}-\frac{1}{25} \exp \{18 \lambda\}$. We may choose $\lambda=0.16$, then $M \approx 11.511<12.932$ $=\exp \{16 \lambda\}$. By Theorem 3.1, the equilibrium point $\left(\frac{\pi}{2}, \pi\right)$ of (3.1) is globally exponentially stable with the approximate convergence rate 0.007 .

Remark 3.2. It can be easily verified that (iv), (v) in Theorem 2 in [22] are violated in the above example. Thus, our results improve the results in [22].

\section{A new inequality}

In this section, we shall give a new inequality that is different from Lemma 1.2 and can be applied to the case not covered in $[19,20]$.

Theorem 4.1. Suppose that

(i) $\alpha>\beta \cdot \max _{k \in \mathbb{Z}_{+}}\left\{\frac{1}{a_{k}+b_{k}}, 1\right\}$;

(ii) $t_{k}-t_{k-1}>\tau$, and there exist constants $M>0, \gamma \geq 0$ such that

$$
\prod_{s=1}^{k}\left(a_{s}+b_{s} \exp \{\lambda \tau\}\right) \leq M \exp \left\{\gamma\left(t_{k}-t_{0}\right)\right\}, \quad k \in \mathbb{Z}_{+},
$$

where $\lambda>0$ satisfies

$$
\lambda \leq \alpha-\beta \max _{k \in \mathbb{Z}_{+}}\left\{\frac{1}{a_{k}+b_{k} \exp \{\lambda \tau\}}, 1\right\} \exp \{\lambda \tau\} .
$$


Then,

$$
f(t) \leq M \bar{f}\left(t_{0}\right) \exp \left\{-(\lambda-\gamma)\left(t-t_{0}\right)\right\}, \quad t \geq t_{0} .
$$

Proof. Condition (i) implies that there exists small enough $\lambda>0$ such that the inequality (4.1) holds.

Next, we show

$$
f(t) \leq \bar{f}\left(t_{0}\right)\left(\prod_{s=0}^{k}\left(a_{s}+b_{s} \exp \{\lambda \tau\}\right)\right) \exp \left\{-\lambda\left(t-t_{0}\right)\right\}, \quad t \in\left[t_{k}, t_{k+1}\right)
$$

where $a_{0}=1, b_{0}=0$.

It is clear that $f(t) \leq \bar{f}\left(t_{0}\right)$ for $t \in\left[t_{0}-\tau, t_{0}\right]$ by the definition of $\bar{f}$.

Take $k=0$, we shall show, for $t \in\left[t_{0}, t_{1}\right)$

$$
f(t) \leq \bar{f}\left(t_{0}\right) \exp \left\{-\lambda\left(t-t_{0}\right)\right\} .
$$

Suppose on the contrary, then there exists some $t \in\left[t_{0}, t_{1}\right)$ such that $f(t)>\bar{f}\left(t_{0}\right) \exp \left\{-\lambda\left(t-t_{0}\right)\right\}$.

Let

$$
t^{\star}=\inf \left\{t \in\left[t_{0}, t_{1}\right), f(t)>W_{0}(t)\right\}, W_{0}(t)=\bar{f}\left(t_{0}\right) \exp \left\{-\lambda\left(t-t_{0}\right)\right\}
$$

then $t^{\star} \in\left[t_{0}, t_{1}\right)$ and

(1) $f\left(t^{\star}\right)=W_{0}\left(t^{\star}\right)$

(2) $f(t) \leq W_{0}(t), t \in\left[t_{0}, t^{\star}\right]$;

(3) $D^{+} f\left(t^{\star}\right)>W_{0}^{\prime}\left(t^{\star}\right)$.

Since $\bar{f}\left(t^{\star}\right)=\sup _{s \in\left[t^{\star}-\tau, t^{\star}\right]} f(s), t^{\star} \in\left[t_{0}, t_{1}\right)$, we get

$$
\bar{f}\left(t^{\star}\right) \leq \bar{f}\left(t_{0}\right) \exp \left\{-\lambda\left(t^{\star}-\tau-t_{0}\right)\right\} .
$$

Hence, we have

$$
\begin{aligned}
D^{+} f\left(t^{\star}\right) & \leq-\alpha f\left(t^{\star}\right)+\beta \bar{f}\left(t^{\star}\right) \\
& \leq-\alpha f\left(t^{\star}\right)+\beta \bar{f}\left(t_{0}\right) \exp \left\{-\lambda\left(t^{\star}-\tau-t_{0}\right)\right\} \\
& \leq-\alpha W_{0}\left(t^{\star}\right)+\beta W_{0}\left(t^{\star}-\tau\right) \\
& \leq-\alpha W_{0}\left(t^{\star}\right)+\beta \max _{k \in \mathbb{Z}_{+}}\left\{\frac{1}{a_{k}+b_{k} \exp \{\lambda \tau\}}, 1\right\} W_{0}\left(t^{\star}-\tau\right) .
\end{aligned}
$$

Thus, by the definitions of $\lambda$ and $W_{0}$, we have

$$
\begin{aligned}
W^{\prime}{ }_{0}\left(t^{\star}\right) & =-\lambda \bar{f}\left(t_{0}\right) \exp \left\{-\lambda\left(t^{\star}-t_{0}\right)\right\} \\
& \geq\left(\beta \max _{k \in \mathbb{Z}_{+}}\left\{\frac{1}{a_{k}+b_{k} \exp \{\lambda \tau\}}, 1\right\} \exp \{\lambda \tau\}-\alpha\right) \bar{f}\left(t_{0}\right) \exp \left\{-\lambda\left(t^{\star}-t_{0}\right)\right\} \\
& =-\alpha \bar{f}\left(t_{0}\right) \exp \left\{-\lambda\left(t^{\star}-t_{0}\right)\right\}+\beta \max _{k \in \mathbb{Z}_{+}}\left\{\frac{1}{a_{k}+b_{k} \exp \{\lambda \tau\}}, 1\right\} \bar{f}\left(t_{0}\right) \exp \left\{-\lambda\left(t^{\star}-\tau-t_{0}\right)\right. \\
& =-\alpha W_{0}\left(t^{\star}\right)+\beta \max _{k \in \mathbb{Z}_{+}}\left\{\frac{1}{a_{k}+b_{k} \exp \{\lambda \tau\}}, 1\right\} W_{0}\left(t^{\star}-\tau\right) \\
& \geq D^{+} f\left(t^{\star}\right),
\end{aligned}
$$

which contradicts (3). So we get that (4.2) holds for all $t \in\left[t_{0}, t_{1}\right)$. 
Now, we assume that for $t \in\left[t_{m-1}, t_{m}\right), m \in \mathbb{Z}_{+}$

$$
f(t) \leq \bar{f}\left(t_{0}\right)\left(\prod_{s=0}^{m-1}\left(a_{s}+b_{s} \exp \{\lambda \tau\}\right)\right) \exp \left\{-\lambda\left(t-t_{0}\right)\right\}
$$

We shall show that for $t \in\left[t_{m}, t_{m+1}\right), m \in \mathbb{Z}_{+}$

$$
f(t) \leq \bar{f}\left(t_{0}\right)\left(\prod_{s=0}^{m}\left(a_{s}+b_{s} \exp \{\lambda \tau\}\right)\right) \exp \left\{-\lambda\left(t-t_{0}\right)\right\}
$$

By (4.3) and the fact that $t_{m}-t_{m-1}>\tau$, we know

$$
\bar{f}\left(t_{m}^{-}\right) \leq \bar{f}\left(t_{0}\right)\left(\prod_{s=0}^{m-1}\left(a_{s}+b_{s} \exp \{\lambda \tau\}\right)\right) \exp \left\{-\lambda\left(t_{m}-\tau-t_{0}\right)\right\}
$$

Hence,

$$
\begin{aligned}
f\left(t_{m}\right) \leq & a_{m} f\left(t_{m^{-}}\right)+b_{m} \bar{f}\left(t_{m}^{-}\right) \\
\leq & a_{m} \bar{f}\left(t_{0}\right)\left(\prod_{s=0}^{m-1}\left(a_{s}+b_{s} \exp \{\lambda \tau\}\right)\right) \exp \left\{-\lambda\left(t_{m}-t_{0}\right)\right\} \\
& +b_{m} \bar{f}\left(t_{0}\right)\left(\prod_{s=0}^{m-1}\left(a_{s}+b_{s} \exp \{\lambda \tau\}\right)\right) \exp \left\{-\lambda\left(t_{m}-\tau-t_{0}\right)\right\} \\
\leq & \left(a_{m}+b_{m} \exp \{\lambda \tau\}\right) \bar{f}\left(t_{0}\right)\left(\prod_{s=0}^{m-1}\left(a_{s}+b_{s} \exp \{\lambda \tau\}\right)\right) \exp \left\{-\lambda\left(t_{m}-t_{0}\right)\right\} \\
\leq & \bar{f}\left(t_{0}\right)\left(\prod_{s=0}^{m}\left(a_{s}+b_{s} \exp \{\lambda \tau\}\right)\right) \exp \left\{-\lambda\left(t_{m}-t_{0}\right)\right\} .
\end{aligned}
$$

If (4.4) is not true, then there exists some $t \in\left[t_{m}, t_{m-1}\right)$ such that

$$
f(t)>\bar{f}\left(t_{0}\right)\left(\prod_{s=0}^{m}\left(a_{s}+b_{s} \exp \{\lambda \tau\}\right)\right) \exp \left\{-\lambda\left(t-t_{0}\right)\right\} .
$$

By (4.5), we define

$$
t^{*}=\inf \left\{t \in\left[t_{m}, t_{m+1}\right), f(t)>W_{m}(t)\right\},
$$

where

$$
W_{m}(t)=\bar{f}\left(t_{0}\right)\left(\prod_{s=0}^{m}\left(a_{s}+b_{s} \exp \{\lambda \tau\}\right)\right) \exp \left\{-\lambda\left(t-t_{0}\right)\right\},
$$

then $t^{*} \in\left[t_{m}, t_{m+1}\right)$ and

(4) $f\left(t^{*}\right)=W_{m}\left(t^{*}\right)$;

(5) $f(t) \leq W_{m}(t), t \in\left[t_{m}, t^{*}\right]$;

(6) $D^{+} f\left(t^{*}\right)>W_{m}^{\prime}\left(t^{*}\right)$.

Since $\bar{f}\left(t^{\star}\right)=\sup _{s \in\left[t^{\star}-\tau, t^{\star}\right]} f(s)_{n}, t^{*} \in\left[t_{m}, t_{m+1}\right)$, we get 


$$
\bar{f}\left(t^{*}\right) \leq W_{m}\left(t^{*}-\tau\right) \max \left\{1, \frac{1}{a_{m}+b_{m} \exp \{\lambda \tau\}}\right\} .
$$

In fact, when $t^{*}-\tau \geq t_{m}$, from (5), we have

$$
\begin{aligned}
\bar{f}\left(t^{*}\right) & \leq \bar{f}\left(t_{0}\right)\left(\prod_{s=0}^{m}\left(a_{s}+b_{s} \exp \{\lambda \tau\}\right)\right) \exp \left\{-\lambda\left(t^{*}-\tau-t_{0}\right)\right\} \\
& \leq W_{m}\left(t^{*}-\tau\right) \\
& \leq W_{m}\left(t^{*}-\tau\right) \max \left\{1, \frac{1}{a_{m}+b_{m} \exp \{\lambda \tau\}}\right\} .
\end{aligned}
$$

When $t^{*}-\tau<t_{m}$, note that $t_{k}-t_{k-1}>\tau$, we have

$$
\begin{aligned}
\bar{f}\left(t^{*}\right) \leq & \max \left\{\bar{f}\left(t_{0}\right)\left(\prod_{s=0}^{m}\left(a_{s}+b_{s} \exp \{\lambda \tau\}\right)\right) \exp \left\{-\lambda\left(t_{m}-t_{0}\right)\right\},\right. \\
& \left.\bar{f}\left(t_{0}\right)\left(\prod_{s=0}^{m-1}\left(a_{s}+b_{s} \exp \{\lambda \tau\}\right)\right) \exp \left\{-\lambda\left(t^{*}-\tau-t_{0}\right)\right\}\right\} \\
\leq & \max \left\{\bar{f}\left(t_{0}\right)\left(\prod_{s=0}^{m}\left(a_{s}+b_{s} \exp \{\lambda \tau\}\right)\right) \exp \left\{-\lambda\left(t^{*}-\tau-t_{0}\right)\right\},\right. \\
& \left.\bar{f}\left(t_{0}\right)\left(\prod_{s=0}^{m-1}\left(a_{s}+b_{s} \exp \{\lambda \tau\}\right)\right) \exp \left\{-\lambda\left(t^{*}-\tau-t_{0}\right)\right\}\right\} \\
\leq & \bar{f}\left(t_{0}\right)\left(\prod_{s=0}^{m}\left(a_{s}+b_{s} \exp \{\lambda \tau\}\right)\right) \exp \left\{-\lambda\left(t^{*}-\tau-t_{0}\right)\right\} \max \left\{1, \frac{1}{a_{m}+b_{m} \exp \{\lambda \tau\}}\right\} \\
\leq & W_{m}\left(t^{*}-\tau\right) \max \left\{1, \frac{1}{a_{m}+b_{m} \exp \{\lambda \tau\}}\right\} .
\end{aligned}
$$

This, together with (4), leads to

$$
\begin{aligned}
D^{+} f\left(t^{*}\right) & \leq-\alpha f\left(t^{*}\right)+\beta \bar{f}\left(t^{*}\right) \\
& \leq-\alpha W_{m}\left(t^{*}\right)+\beta W_{m}\left(t^{*}-\tau\right) \max \left\{1, \frac{1}{a_{m}+b_{m} \exp \{\lambda \tau\}}\right\} .
\end{aligned}
$$

Hence, we obtain

$$
\begin{aligned}
W_{m}^{\prime}\left(t^{*}\right)= & -\lambda \bar{f}\left(t_{0}\right)\left(\prod_{s=0}^{m}\left(a_{s}+b_{s} \exp \{\lambda \tau\}\right)\right) \exp \left\{-\lambda\left(t^{*}-t_{0}\right)\right\} \\
\geq & \left(\beta \max _{k \in \mathbb{Z}_{+}}\left\{\frac{1}{a_{k}+b_{k} \exp \{\lambda \tau\}}, 1\right\} \exp \{\lambda \tau\}-\alpha\right) \bar{f}\left(t_{0}\right) \\
& \times\left(\prod_{s=0}^{m}\left(a_{s}+b_{s} \exp \{\lambda \tau\}\right)\right) \exp \left\{-\lambda\left(t^{*}-t_{0}\right)\right\} \\
\geq & -\alpha W_{m}\left(t^{*}\right)+\beta \max \left\{1, \frac{1}{a_{m}+b_{m} \exp \{\lambda \tau\}}\right\} W_{m}\left(t^{*}-\tau\right) \\
\geq & D^{+} f\left(t^{*}\right),
\end{aligned}
$$

which is a contradiction with (6). Hence, we obtain (4.4) holds for all $t \in\left[t_{m}, t_{m+1}\right)$, $m \in \mathbb{Z}_{+}$. Thus, by the method of induction, we get, for $t \in\left[t_{k}, t_{k+1}\right)$ 


$$
f(t) \leq \bar{f}\left(t_{0}\right)\left(\prod_{s=0}^{k}\left(a_{s}+b_{s} \exp \{\lambda \tau\}\right)\right) \exp \left\{-\lambda\left(t-t_{0}\right)\right\}, \quad k \in \mathbb{Z}_{+} .
$$

By condition (ii), we have

$$
f(t) \leq M \bar{f}\left(t_{0}\right) \exp \left\{-(\lambda-\gamma)\left(t-t_{0}\right)\right\}, t \geq t_{0},
$$

where $\lambda$ satisfies (4.1). The proof of Theorem 4.1 is therefore completed. $\square$

Remark 4.1. If there exists constant $M>0$ such that $\prod_{s=1}^{k}\left(a_{s}+b_{s} \exp \{\lambda \tau\}\right) \leq M$ for all $k \in \mathbb{Z}_{+}$holds, then we can choose $\gamma=0$ in Theorem 4.1.

If let $b_{k}=0, k \in \mathbb{Z}_{+}$in Theorem 4.1, then we can obtain the following result.

\section{Corollary 4.1. Suppose that}

(iii) $\alpha>\beta \cdot \max _{k \in \mathbb{Z}_{+}}\left\{\frac{1}{a_{k}}, 1\right\}$;

(iv) $t_{k}-t_{k-1}>\tau$, and there exist constants $M>0, \gamma \geq 0$ such that

$$
\prod_{s=1}^{k} a_{s} \leq M \exp \left\{\gamma\left(t_{k}-t_{0}\right)\right\}, \quad k \in \mathbb{Z}_{+}
$$

where $\lambda>0$ satisfies

$$
\lambda \leq \alpha-\beta \max _{k \in \mathbb{Z}_{+}}\left\{\frac{1}{a_{k}}, 1\right\} \exp \{\lambda \tau\}
$$

Then,

$$
f(t) \leq M \bar{f}\left(t_{0}\right) \exp \left\{-(\lambda-\gamma)\left(t-t_{0}\right)\right\}, \quad t \geq t_{0} .
$$

In the following, the superiority of the present approach over $[19,20]$ will be demonstrated by an example. The main tool for studying the neural network in $[19,20]$ is the following:

Lemma 4.1. Suppose that $\alpha>\beta \geq 0$, and $f(t)$ satisfies scalar impulsive differential inequality

$$
\begin{cases}D^{+} f(t) \leq-\alpha f(t)+\beta \bar{f}(t), & t \neq t_{k}, \\ f\left(t_{k}\right) \leq a_{k} f\left(t_{k^{-}}\right), & k \in \mathbb{Z}_{+},\end{cases}
$$

where

$$
f(t) \geq 0, \quad \bar{f}(t)=\sup _{s \in[t-\tau, t]} f(s), \quad \bar{f}\left(t^{-}\right)=\sup _{s \in[t-\tau, t)} f(s),
$$

and $f(t)$ is continuous except at each $t_{k}, k \in \mathbb{Z}_{+}$, where it has jump discontinuities. The sequence $\left\{t_{k}\right\}$ satisfies $0 \leq t_{0}<t_{1}<\ldots<t_{k}<\ldots, \lim _{k \rightarrow+\infty} t_{k}=+\infty$.

Then,

$$
f(t) \leq \bar{f}\left(t_{0}\right)\left(\prod_{t_{0} \leq t_{k} \leq t} \max \left\{1,\left|a_{k}\right|\right\}\right) \exp \left\{-\lambda\left(t-t_{0}\right)\right\}, \quad k \in \mathbb{Z}_{+},
$$


where

$$
\lambda \leq \alpha-\beta \exp \{\lambda \tau\}
$$

Consider a particular network of two neurons as follows:

$$
\left\{\begin{array}{l}
x^{\prime}(t)=-4.6 x(t)+0.6 \sin x(t)-0.5 \sin \gamma\left(t-\tau_{1}\right), \quad t \neq t_{k}, \\
y^{\prime}(t)=-5 y(t)+0.4 \cos \gamma(t)-0.4 \cos x\left(t-\tau_{2}\right), \quad t \neq t_{k} \\
x\left(t_{k}\right)=\beta_{k} x\left(t_{k}^{-}\right), y\left(t_{k}\right)=\gamma_{k} y\left(t_{k}^{-}\right), k \in \mathbb{Z}_{+}
\end{array}\right.
$$

where $t_{k}-t_{k-1}=0.25, t_{0}=0, k \in \mathbb{Z}_{+}, \tau_{i} \in(0,0.25), i=1,2$ and

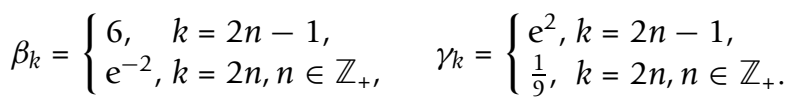

Let $\tau=\max \left\{\tau_{1}, \tau_{2}\right\}$, then $\tau \in(0,0.25)$.

Choose $V(t)=|x(t)|+|y(t)|$, then

$$
\begin{aligned}
\left.D^{+} V\right|_{(4.7) \leq} & -4.6|x(t)|+0.6|\sin x(t)|+0.5\left|\sin \gamma\left(t-\tau_{1}\right)\right|-5|\gamma(t)| \\
& +0.4|\cos \gamma(t)|+0.4\left|\cos x\left(t-\tau_{2}\right)\right| \\
\leq & -4|x(t)|+0.5\left|\gamma\left(t-\tau_{1}\right)\right|-4.6|\gamma(t)|+0.4\left|x\left(t-\tau_{2}\right)\right| \\
\leq & -4[|x(t)|+|\gamma(t)|]+0.5\left[\left|\gamma\left(t-\tau_{1}\right)\right|+\left|x\left(t-\tau_{2}\right)\right|\right] \\
\leq & -4 V(t)+0.5 \tilde{V}(t)
\end{aligned}
$$

where $\widetilde{V}(t)=\sup _{t-\tau \leq s \leq t} V(s)$.

Moreover,

$$
V\left(t_{k}\right)=\left|x\left(t_{k}\right)\right|+\left|\gamma\left(t_{k}\right)\right| \leq \max \left\{\beta_{k}, \gamma_{k}\right\}\left[\left|x\left(t_{k}^{-}\right)\right|+\left|\gamma\left(t_{k}^{-}\right)\right|\right],
$$

where

$$
\max \left\{\beta_{k}, \gamma_{k}\right\}=\left\{\begin{array}{l}
e^{2}, k=2 n-1, \\
e^{-2} k=2 n, n \in \mathbb{Z}_{+},
\end{array}\right.
$$

Choose $M=\mathrm{e}^{2}, \gamma=0$ in Corollary 4.1, we get

$$
|x(t)|+|\gamma(t)|=V(t) \leq \mathrm{e}^{2} \widetilde{V}\left(t_{0}\right) \exp \left\{-\lambda\left(t-t_{0}\right)\right\},
$$

where $\lambda>0$ satisfies $\lambda \leq 4-0.5 \mathrm{e}^{2} \exp \{\lambda \tau\}$. Hence, the equilibrium point $(0,0)$ of (4.7) is globally exponentially stable with the approximate convergence rate $\lambda$.

On the other hand, we will point out the inequality (4.6) is not feasible here.

In fact, by using the inequality (4.6), we get, for $t \in\left[t_{k}, t_{k+1}\right)$,

$$
\begin{aligned}
|x(t)|+|y(t)|=V(t) & \leq \widetilde{V}\left(t_{0}\right)\left(\mathrm{e}^{2}\right)^{\frac{k+1}{2}} \exp \left\{-\lambda\left(t-t_{0}\right)\right\} \\
& \leq \widetilde{V}\left(t_{0}\right) \mathrm{e}^{k+1} \mathrm{e}^{-\frac{\lambda k}{4}} \\
& \leq \widetilde{V}\left(t_{0}\right)\left(\mathrm{e}^{1-\frac{\lambda}{4}}\right)^{k} \mathrm{e} \rightarrow+\infty \text { as } t \rightarrow \infty,
\end{aligned}
$$

since $\lambda>0$ satisfies $\lambda \leq 4-0.5 \exp \{\lambda \tau\}$. This leads to that it is very difficult to get the estimation formula like (4.8). Therefore, our method is less conservative in some degree than that in $[19,20]$. 


\section{Competing interests}

The author declares that he has no competing interests.

Received: 17 May 2011 Accepted: 31 October 2011 Published: 31 October 2011

\section{References}

1. Newman, M: The structure and function of complex networks. SIAM Rev. 45, 167-256 (2003). doi:10.1137/ S003614450342480

2. Gopalsamy, K, He, X: Stability in asymmetric Hopfield nets with transmission delays. Phys D. 76, 344-358 (1994). doi:10.1016/0167-2789(94)90043-4

3. Cao, J, Chen, A, Huang, X: Almost periodic attractor of delayed neural networks with variable coefficients. Phys Lett A. 340, 104-120 (2005). doi:10.1016/j.physleta.2005.04.021

4. Gui, Z, Ge, W: Existence and uniqueness of periodic solutions of nonautonomous cellular neural networks with impulses. Phys Lett A. 354, 84-94 (2006). doi:10.1016/j.physleta.2006.01.018

5. Lou, X, Cui, B: Novel global stability criteria for high-order Hopfield-type neural networks with time-varying delays. J Math Anal Appl. 330, 144-158 (2007). doi:10.1016/j.jmaa.2006.07.058

6. Zhang, Q, Wei, X, Xu, J: Delay-dependent global stability condition for delayed Hopfield neural networks. Nonlinear Anal. 8, 997-1002 (2007). doi:10.1016/j.nonrwa.2006.05.002

7. Liu, B: Almost periodic solutions for Hopfield neural networks with continuously distributed delays. Math Comput Simul. 73, 327-335 (2007). doi:10.1016/j.matcom.2006.05.027

8. Liu, B, Huang, L: Existence and exponential stability of almost periodic solutions for Hopfield neural networks with delays. Neurocomputing. 68, 196-207 (2005)

9. Chen, Z, Ruan, J: Global stability analysis of impulsive Cohen-Grossberg neural networks with delay. Phys Lett A. 345 101-111 (2005). doi:10.1016/j.physleta.2005.06.104

10. Arik, S, Tavsanoglu, V: On the global asymptotic stability of delayed cellular neural networks. IEEE Trans Circuits Syst I. 47(4), 571-574 (2000). doi:10.1109/81.841859

11. Arik, S, Tavsanoglu, V: Global asymptotic stability analysis of bidirectional associative memory neural networks with constant time delays. Neurocomputing. 68, 161-176 (2005)

12. Feng, C, Plamondon, R: Stability analysis of bidirectional associative memory networks with time delays. IEEE Trans Neural Netw. 14, 1560-1565 (2003). doi:10.1109/TNN.2003.820829

13. Zhang, Y, Sun, J: Stability of impulsive neural networks with time delays. Phys Lett A. 348, 44-50 (2005). doi:10.1016/j. physleta.2005.08.030

14. Liu, X, Teo, K, Xu, B: Exponential stability of impulsive high-order Hopfield-type neural networks with time-varying delays. IEEE Trans Neural Netw. 16, 1329-1339 (2005). doi:10.1109/TNN.2005.857949

15. Qiu, J: Exponential stability of impulsive neural networks with time-varying delays and reaction-diffusion terms. Neurocomputing. 70, 1102-1108 (2007)

16. Yang, Z, Xu, D: Global exponential stability of Hopfield neural networks with variable delays and impulsive effects. Appl Math Mech. 27(11), 1517-1522 (2006). doi:10.1007/s10483-006-1109-1

17. Akca, H., et al: Continuous-time additive Hopfield-type neural networks with impulses. J Math Anal Appl. 290, 436-451 (2004). doi:10.1016/j.jmaa.2003.10.005

18. Yue, D, Xu, S, Liu, Y: Differential inequality with delay and impulse and its applications to design robust control. Control Theory Appl. 16(4), 519-524 (1999)

19. Zhou, J, Xiang, L, Liu, Z: Synchronization in complex delayed dynamical networks with impulsive effects. Physica A. 384 , 684-692 (2007). doi:10.1016/j.physa.2007.05.060

20. Yang, Z, Xu, D: Stability analysis of delay neural networks with impulsive effects. IEEE Trans Circuits Syst I. 52(1), 517-521 (2005)

21. $\mathrm{Xu}, \mathrm{D}$, Yang, Z: Impulsive delay differential inequality and stability of neural networks. J Math Anal Appl. 305, 107-120 (2005). doi:10.1016/j.jmaa.2004.10.040

22. Yang, F, Zhang, C, Wu, D: Global stability analysis of impulsive BAM type Cohen-Grossberg neural networks with delays. Appl Math Comput. 186, 932-940 (2007). doi:10.1016/j.amc.2006.08.016

23. Cao, J, Wang, J: Global asymptotic stability of a general class of recurrent neural networks with time-varying delays. IEEE Trans Circuits Syst I. 50, 34-44 (2003). doi:10.1109/TCSI.2002.807494

24. Guo, S, Huang, L: Stability analysis of a delayed Hopfield neural network. Phys Rev E. 67, 119-122 (2003)

25. Li, X: Existence and global exponential stability of periodic solution for impulsive Cohen Grossberg-type BAM neural networks with continuously distributed delays. Appl Math Comput. 215, 292-307 (2009). doi:10.1016/j.amc.2009.05.005

26. Pan, L, Cao, J: Oscillations of even order linear impulsive delay differential equations. Differ Equ Appl. 2, 163-176 (2010)

27. Ho, DWC, Liang, J, Lam, J: Global exponential stability of impulsive high-order BAM neural networks with time-varying delays. Neural Netw. 19, 1581-1590 (2006). doi:10.1016/j.neunet.2006.02.006

28. Lu, J, Ho, DWC, Cao, J: A unified synchronization criterion for impulsive dynamical networks. Automatica. 46, 1215-1221 (2010). doi:10.1016/j.automatica.2010.04.005

doi:10.1186/1029-242X-2011-103

Cite this article as: Wang: Further analysis on stability of delayed neural networks via inequality technique.

Journal of Inequalities and Applications 2011 2011:103. 